\title{
Combined Measurement of the Inclusive Diffractive Cross Section at HERA
}

\section{Marta Ruspa*t}

Univ. Piemonte Orientale, Novara and INFN, Torino, Italy

E-mail: ruspa@to.infn.it

\begin{abstract}
A combination is presented of the inclusive diffractive cross section measurements made by the H1 and ZEUS Collaborations at HERA. The analysis uses diffractive deep inelastic scattering data selected by means of proton spectrometers. Correlations of systematic uncertainties are taken into account by the combination method, resulting in improved precision.
\end{abstract}

The 2011 Europhysics Conference on High Energy Physics-HEP 2011,

July 21-27, 2011

Grenoble, Rhône-Alpes France

*Speaker.

${ }^{\dagger}$ For the H1 and ZEUS Collaborations 


\section{Inclusive Diffraction at HERA}

In the single diffractive dissociation process in proton-proton scattering, $p p \rightarrow X p$, at least one of the beam hadrons emerges intact from the collision, having lost only a small fraction of its energy and gained only a small transverse momentum. In the analogous process involving virtual photons, $\gamma^{\star} p \rightarrow X p$, an exchanged photon of virtuality $Q^{2}$ dissociates through its interaction with the proton to produce a hadronic system $X$ with mass $M_{X}$. The fractional longitudinal momentum loss of the proton during the interaction is denoted $x_{P}$, while the fraction of this momentum carried by the struck quark is denoted $\beta$. These variables are related to Bjorken $x$ by $x=x_{P} \beta$.

Diffractive processes have been studied extensively in deep-inelastic ep scattering (DIS) by the H1 and ZEUS experiments at HERA. A recent review can be found in [1].

Because of the variety of the selection methods, the complementary features of the data and the statistical limitations of some samples, combining the H1 and ZEUS data can provide the most precise and kinematically extended data on diffractive cross sections in DIS, which can eventually be used to extract diffractive parton distribution functions. A first step, presented here, is taken towards such long term perspective by combining the H1 [2] and the ZEUS [3] measurements obtained with dedicated proton spectrometers movable very close to the beam line to tag the scattered proton (the Forward Proton Spectrometer, FPS, for H1 and the Leading Proton Spectrometer, LPS, for ZEUS).

\section{Combination method}

The H1 FPS data [2] correspond to an integrated luminosity of $156.6 \mathrm{pb}^{-1}$ and were collected in the years from 2005 to 2007, after the HERA luminosity upgrade. The ZEUS LPS sample [3] was collected in the years 1999 and 2000 and corresponds to an integrated luminosity of 32.6 $\mathrm{pb}^{-1}$. Since the H1 and ZEUS measurements were based on a different binning, the ZEUS points were swum to the H1 bin centers by using the NLO QCD fit ZEUS SJ [4].

In the analyses [2] and [3] the cross sections are directly measured for ranges of the squared four-momentum transfer $t$ visible to the proton taggers and extrapolated to the full range $0<|t|<1$ $\mathrm{GeV}^{2}$, assuming for the $t$ dependence of the diffractive cross section a single exponential and using the exponential slope measured from the data. The extrapolation introduces an extra normalization uncertainty. To avoid such systematic effect the $\mathrm{H} 1$ and ZEUS data were combined in the restricted $t$ range $0.09<|t|<0.55 \mathrm{GeV}^{2}$, common to both acceptances. In such range the ratio of the $\mathrm{H} 1$ FPS to the ZEUS LPS data, averaged over the measured kinematic range, is $0.91 \pm 0.01$ (stat) \pm 0.03 (sys) \pm 0.08 (norm), closer to 1 than in the published $t$ range [2]. The resulting kinematic coverage of the combined data is $0.09<|t|<0.55 \mathrm{GeV}^{2}, 2.5<Q^{2}<200 \mathrm{GeV}^{2}, 0.0018<\beta<0.56$ and $0.0009<x_{\mathbb{P}}<0.09$.

The combination method is based on an iterative $\chi^{2}$ minimization procedure already used for previous HERA combined results $[5,6]$. The only theoretical input of such averaging procedure is the assumption that the $\mathrm{H} 1$ and ZEUS experiments are measuring the same cross section at the same kinematic points. The correlated systematic uncertainties are floated coherently so that the two experiments effectively calibrate each other. This allows a model independent check of the data consistency and a significant reduction of the correlated systematic uncertainty. 


\section{Results}

In the minimization procedure 227 data points were combined to 169 cross section measurements. The data show good consistency with $\chi^{2} / n d o f=52 / 58$. In Fig. 1 the combined data are compared to the input H1 FPS and ZEUS LPS data. The combination is driven by the H1 results, which are statistically more powerful. The combined measurement shows though an average improvement in precision of about $20 \%$ with respect to the original $\mathrm{H} 1$ data.
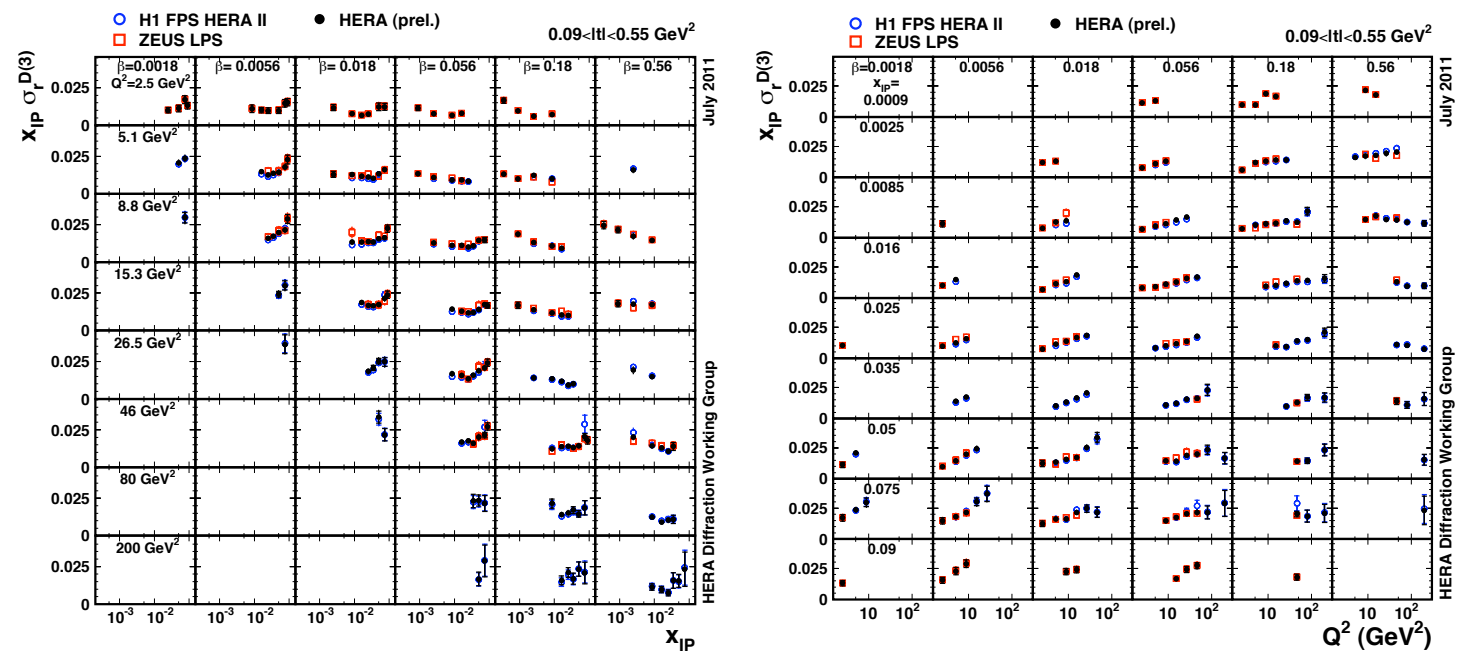

Figure 1: HERA diffractive reduced cross section as a function of $x_{\mathbb{P}}$ for different $\beta$ and $Q^{2}$ (left) and as a function of $Q^{2}$ for different $\beta$ and $x_{\mathbb{P}}$, compared to the H1 FPS [2] and the ZEUS LPS [3] measurements.

A total of 20 sources of correlated systematic uncertainties were considered, which shift by up to $1 \sigma$ of the nominal value in the averaging procedure, with the exception of the H1 hadronic energy scale which shifts by $1.6 \sigma$. The influence of several correlated systematic uncertainties is reduced significantly.

These combined data are very valuable in the scenario of inclusive diffraction at HERA and beyond. They can provide the absolute normalization of the diffractive cross section in DIS and can help to quantify background contributions in the samples selected with the requirement of a gap in rapidity between the proton and the system $X$ (LRG technique $[3,7,8]$ ).

\section{References}

[1] P. Newman and M. Ruspa, arXiv:0903.2957v1[hep-ex].

[2] H1 Coll., A. Atkas et al., Eur. Phys. J. C 48 (2006) 749.

[3] ZEUS Coll., S. Chekanov et al., Nucl. Phys. B 816 (2009) 1.

[4] ZEUS Coll., S. Chekanov et al., Nucl. Phys. B 831 (2010) 1.

[5] H1 Coll., F.D. Aaron et al., Eur. Phys. J. C 63 (2009) 625.

[6] H1 and ZEUS Coll., F.D. Aaron et al., JHEP 01 (2010) 109.

[7] H1 Coll., A. Aktas et al., Eur. Phys. J. C 48 (2006) 715.

[8] H1 Coll., F.D. Aaron et al., H1prelim-10-011. 